\title{
Acute hospital at home: a new age
}

\author{
Authors: James Richards, Patricia McCormack, Paul Andrews and Peter Szedelac
}

\section{Aims}

We aim to assess the effectiveness and impact of the Acute Hospital at Home (AHAH) service at Dorset County Hospital.

\section{Methods}

The AHAH service has 15 beds, and can be seen as a virtual inpatient ward. Patients are transferred from inpatient care to under the umbrella of AHAH. This is treated as a ward transfer, rather than discharge from hospital.

Each patient is reviewed by a member of the medical team, and then assessed for suitability by members of the AHAH nursing and therapy team.

A number of aspects of inpatient care are then undertaken in the patient's own home, including:

$>$ nursing assessments and observations

$>$ blood tests

$>$ intravenous antibiotics and diuretics

nebulisers

$>$ physiotherapy, occupational and speech and language therapy, together with input from dieticians.

There is a medical review via a multidisciplinary meeting, including review of the patient's history and clinical progress, observations, blood tests and medications.Patients requiring a direct medical assessment are then seen in their own home, or if they require further tests, such as radiographs or CT scans, they are managed through the acute medical unit's ambulatory care service.

\section{Results}

Between January 2014 and January 2015, 275 patients have been referred for assessment by the AHAH team, and 223 have been managed through the service.

An average of 7 bed-days have been saved each day. The four most common conditions managed by the service are cellulitis (12.5\%), exacerbations of COPD and bronchiectasis (10\%), venous thromboembolism (6\%), and diabetic foot ulcers requiring intravenous antibiotics $(3.5 \%)$.

One of the key factors of the service has been the degree of patient satisfaction: $99.3 \%$ of patients, in the friends and family test, were 'likely or extremely likely to recommend the service'.

\section{Conclusions}

The AHAH service at Dorset County Hospital has been a success, and has exceeded the original aim of saving 4 inpatient bed-days each day. The service has provided medical and nursing care together with therapy in people's own homes, reducing the risk of hospital-acquired infection, venous thromboembolism, poor sleep in crowded inpatient wards and deconditioning.

The three key factors in the success of AHAH are the provision of aspects of inpatient care at home, the multidisciplinary nature of the service, and the high degree of patient satisfaction. 